\title{
Probing a four flavor vis-a-vis three flavor neutrino mixing for ultrahigh energy neutrino signals at a $1 \mathrm{~km}^{2}$ detector
}

\author{
Madhurima Pandey $^{*}$ and Debasish Majumdar ${ }^{\dagger}$ \\ Astroparticle Physics and Cosmology Division, \\ Saha Institute of Nuclear Physics, HBNI 1/AF Bidhannagar, Kolkata 700064, India \\ Amit Dutta Banik \\ Department of Physics, Indian Institute of Technology, Guwahati 781039, India
}

(Received 27 December 2017; published 25 May 2018)

\begin{abstract}
We consider a four-flavor scenario for the neutrinos where an extra sterile neutrino is introduced to the three families of active neutrinos and study the deviation from the three-flavor scenario in the ultrahighenergy (UHE) regime. We calculate the possible muon and shower yields at a $1 \mathrm{~km}^{2}$ detector such as IceCube for these neutrinos from distant UHE sources, e.g., gamma-ray bursts, etc. Similar estimations for muon and shower yields are also obtained for the three-flavor case. Comparing the two results, we find considerable differences between the yields for these two cases. This can be useful for probing the existence of a fourth sterile component using UHE neutrino flux.
\end{abstract}

DOI: 10.1103/PhysRevD.97.103015

\section{INTRODUCTION}

Numerous experiments have established that neutrinos occur in three active flavors. But the existence of a fourth sterile neutrino was proposed long ago, and this possibility has been pursued for quite some time. The neutrino oscillation data from experiments like the Liquid Scintillator Neutrino Detector (LSND) [1-3] cannot be satisfactorily explained by the three-neutrino oscillation framework. The observed excess in LSND data is consistent with $\bar{\nu}_{\mu}-\bar{\nu}_{e}$ oscillation with $0.2 \mathrm{eV}^{2} \leq \Delta m^{2} \leq 10 \mathrm{eV}^{2}$. But this mass-squared difference is not consistent with $\Delta m_{21}^{2}$ or $\Delta m_{32}^{2}$ obtained from solar or atmospheric neutrino experiments. This is also substantiated by the analysis of the excess observed by the MiniBooNE experiment for both $\bar{\nu}_{\mu}-\bar{\nu}_{e}$ and $\nu_{\mu}-\nu_{e}$ oscillations [4,5]. These results suggest the existence of an additional fourth neutrino with a mass-squared splitting $\Delta m_{41}^{2} \gg \Delta m_{32}^{2}$. This fourth neutrino, if it exists, will not have other Standard Model couplings as indicated by the LEP experiment's data on the $Z$-boson decay width. Hence, this additional neutrino (if it exists) is referred to as a sterile neutrino. In addition, experiments have reported reactor

\footnotetext{
*madhurima.pandey@saha.ac.in †debasish.majumdar@saha.ac.in *amitdbanik@iitg.ernet.in
}

Published by the American Physical Society under the terms of the Creative Commons Attribution 4.0 International license. Further distribution of this work must maintain attribution to the author(s) and the published article's title, journal citation, and DOI. Funded by SCOAP ${ }^{3}$. neutrino anomalies where lower rates were found for $\overline{\nu_{e}}$ from nuclear reactors at a distance that is too short for any effective neutrino oscillation among standard neutrinos [6-8]. Lower rates have also been observed at $3 \sigma$ for $\nu_{e}$ 's from ${ }^{51} \mathrm{Cr}$ and ${ }^{37} \mathrm{Ar}$ sources in solar neutrino experiments with gallium [9-13].

Several current experiments are analyzing their data for signals of a fourth sterile neutrino, and have given bounds on different oscillation parameters. The MINOS experiment [14] measures $\nu_{\mu}$ oscillations using charged-current (CC) and neutral-current (NC) interactions in a longbaseline experiment with far and near detectors that have a baseline separation of $734 \mathrm{~km}$. The MINOS and upgraded MINOS + experiments have recently put constraints on sterile neutrino oscillation parameters $\left(\sin ^{2} \theta_{24}-\Delta m_{41}^{2}\right)$ $[15,16]$. The NOvA experiment, on the other hand, is another long-baseline $(810 \mathrm{~km})$ neutrino experiment that looks for $\nu_{\mu}-\nu_{s}$ oscillations (with a $\nu_{\mu}$ beam from NuMI at Fermilab) through NC interactions. The NOvA experiment searches for oscillations in the disappearance channel of the active neutrino flux in the near and far detectors.

New data from reactor and other short- and long-baseline neutrino experiments (such as MINOS [14-25], Daya Bay [25-32], Bugey [33], etc.) and their analyses considering the active-sterile neutrino oscillation have given new bounds on active-sterile mixing angles and $\Delta m^{2}$.

There are other future long-baseline experiments, such as the Deep Underground Neutrino Experiment (DUNE) [34-37], T2HK [38-40], etc., that may shed more light on neutrino oscillation physics and enrich the search for activesterile neutrino oscillations. For example, the neutral-current 
data from DUNE (with a long-baseline length of about $1300 \mathrm{~km}$ between the neutrino source at Fermilab and the detector at the Sanford Underground Research Facility in South Dakota) will be useful if active neutrinos oscillate to sterile neutrinos [41].

In this work, we adopt the four $(3+1)$-neutrino scheme where we have three active neutrinos and one sterile neutrino and a four-flavor oscillation scenario instead of the usual three-active-neutrino case. We also separately consider the three-active-neutrino scenario and the threeflavor oscillations. Our purpose is to explore the possibility of an experimental signature that could indicate the existence of a sterile neutrino. To this end, we consider ultrahigh-energy (UHE) neutrinos from distant extragalactic sources and their detection possibilities in a large terrestrial neutrino telescope such as IceCube [42]. Highenergy events such as gamma-ray bursts (GRBs) can produce such neutrinos through their particle acceleration mechanism. GRBs are thought to arise from infalling accreted matter bouncing off of a failed star that has possibly turned into a black hole. In the process, a powerful shock wave progresses outwards with energies as high as $\sim 10^{53}$ ergs or more in the form of a "fireball." The accelerated protons inside such a fireball interact with $\gamma$ 's via a cosmic beam dump, while the pions produced decay to ultrahigh-energy neutrinos. This is commonly known as the fireball (FB) model for GRBs. However, there are also other types of mechanisms such as the cannonball (CB) model which can also explain GRB pulses and the afterglow from core-collapse supernovae [43-46]. In the CB model, it is assumed that an accretion disk is formed around the exploding supernova. When matter from the accretion disk falls into the compact object due to the loss of rotational energy, a pair of cannonballs made up of matter are ejected. Electrons in the cannonballs then undergo Compton scattering with photons to produce GRB energy. Every such CB produced generates a single GRB pulse. On the other hand, in the FB model of GRBs pulses are due to the synchrotron radiation of accreted matter. In the FB mechanism of GRBs afterglows also occur due to synchrotron radiation from the collision of conical outer shells with the interstellar medium (ISM). On the other hand, in the CB model afterglows are initially due to thermal bremsstrahlung when the ejected $\mathrm{CB}$ rapidly expands and interacts with the ISM, and later it is governed by synchrotron radiation [45]. In the present work we perform our calculations for the fireball model of GRBs only.

The UHE neutrinos therefore will ideally be produced from the decay of pions by he GRB process in the ratio $\nu_{e}: \nu_{\mu}: \nu_{\tau}=1: 2: 0$. These neutrinos will suffer flavor oscillations or suppressions while traversing to a terrestrial detector. Because of the astronomical distances between the GRBs and the Earth, the oscillatory part $\left[\sin ^{2}\left(\Delta m^{2}[L / 4 E]\right)\right]$ of the oscillation probability equation averages out ( $L$ and $E$ are the baseline length and energy of the neutrinos, respectively, while $\Delta m^{2}$ denotes the masssquared difference of any two neutrino species). Thus, in the oscillation probability equations one is left with just three oscillation parameters, namely, the three mixing angles $\theta_{12}, \theta_{23}$, and $\theta_{13}$ in the three-active-neutrino scenario. However, in the $(3+1)$ four-neutrino scheme considered here there are three additional mixing angles, namely, $\theta_{14}, \theta_{24}$, and $\theta_{34}$, which account for the mixing of the three active neutrinos with the fourth sterile neutrino. In this work we adopt the experimental best-fit values for the three active neutrino mixing angles $\theta_{12}, \theta_{23}$, and $\theta_{13}$ obtained from the analysis of data from solar neutrinos, atmospheric neutrinos, reactor and accelerator neutrinos, etc. But the active-sterile mixing angles are not known with certainty. However, as discussed earlier in this section, bounds or limits on these unknown mixing angles have been obtained from the analyses of various reactor- or accelerator-based neutrino experiments. With upcoming long-baseline experiments along with the increasing amount of data available from existing experiments, these bounds are expected to be more stringent.

As mentioned earlier, we consider here the UHE neutrinos from GRBs and estimate the possible detection yield at a $1 \mathrm{~km}^{2}$ detector such as IceCube [42] for the fourneutrino $(3+1)$ oscillation scheme. Similar estimations are also made using the usual three-active-neutrino scheme and their oscillations. We consider two kinds of signals, namely, the muon track signal and the shower/cascade that may be produced by the CC and NC interactions of GRB neutrinos during their passage through the Earth or an IceCube-like detector. The muons are obtained when the UHE $\nu_{\mu}$ 's from GRBs reach the Earth and interact with the Earth's interior while moving towards the detector. The CC interactions of $\nu_{\mu}$ and $\nu_{\tau}$ yield $\mu$ and $\tau$, respectively $\left(\nu_{\alpha}+N \rightarrow \alpha+X\right.$, where $\alpha \equiv \mu$ or $\left.\tau\right)$. The muons are detected by the track events in an ice detector through their Cherenkov light. The $\tau$ can be detected via "doublebang" or "lollipop" events. The first bang of "double-bang" events is produced at the site of the first CC interaction $\nu_{\tau}+N \rightarrow \tau+X$ when a $\tau$ track followed by a cascade is generated, and the second bang of the hadronic or electromagnetic shower occurs when $\nu_{\tau}$ is regenerated from the decay of $\tau$ in the fiducial volume of the detector. A lollipop event is one where the first bang could not be detected, but the $\tau$ track can be detected or reconstructed along with the second bang. In the case of an inverse lollipop event, the first bang and the neutrino track can be obtained, while the second bang evades detection. In this work we do not consider these events related to $\nu_{\tau} \mathrm{CC}$ interactions as these detections are not very efficient and could be significant only in an energy window of $\sim 2-10 \mathrm{PeV}$. However, in this work we include in our analysis the muon track signal that can be obtained from $\nu_{\tau}$ via the process $\nu_{\tau} \rightarrow \tau \rightarrow \bar{\nu}_{\mu} \mu \nu_{\tau}$. The CC interactions of $\nu_{e}$ produce electromagnetic showers. Shower events are also considered from the NC 
interactions of neutrinos of all active flavors. The computations for these events are performed for both the $(3+1)$ scheme and the three-active-flavor scheme. We then compare our results for these two scenarios.

We also calculate the effective Majorana $m_{e e}$ for the present $(3+1)$ neutrino (three active and one sterile) framework and obtain its variation with the mass of the lightest neutrino. We then compare our results with the known bounds from the neutrino double-beta decay experiments. We find that for lower masses of the lightest neutrino, the inverted hierarchy of neutrino masses in the $(3+1)$ scenario barely satisfies these limits.

The paper is organized as follows. In Sec. II we briefly discuss the formalism for UHE neutrino fluxes from diffuse GRBs as well as that from a single GRB. This neutrino flux experiences flavor oscillations as it propagates from the GRB sources to the Earth. Neutrino fluxes at the Earth from these high-energy sources (GRBs) are calculated for both the case with three active neutrinos and their oscillations and the case with three active and one sterile neutrino $[(3+1)$ scheme $]$ where a four-neutrino oscillation scenario is considered. Section II is divided into four subsections. Section II A contains the calculation of both the $(3+1)$ flavor and three-flavor neutrino oscillation probabilities, while Sec. II B deals with the UHE neutrino fluxes at the Earth for the four- and three-flavor cases from GRBs. The analytical expressions for the total number of neutrinoinduced muons and shower events from diffuse GRB sources at the $1 \mathrm{~km}^{2}$ IceCube detector are addressed in Sec. II C, while the same from a single GRB are discussed in Sec. II D. The calculational results are discussed in Sec. III for diffuse GRB neutrino fluxes as well as for neutrino fluxes from each of the different single GRBs at given redshifts. The neutrinoless double-beta decay in the $(3+1)$-flavor scenario is given in Sec. IV. Finally, in Sec. V we summarize the paper and provide concluding remarks.

\section{FORMALISM}

\section{A. Four- and three-neutrino oscillations}

In general, the probability for a neutrino $\left|\nu_{\alpha}\right\rangle$ of flavor $\alpha$ to oscillate to a neutrino $\left|\nu_{\beta}\right\rangle$ of flavor $\beta$ is given by [47] (considering no $C P$ violation in the neutrino sector)

$$
P_{\nu_{\alpha} \rightarrow \nu_{\beta}}=\delta_{\alpha \beta}-4 \sum_{j>i} U_{\alpha i} U_{\beta i} U_{\alpha j} U_{\beta j} \sin ^{2}\left(\frac{\pi L}{\lambda_{i j}}\right) .
$$

In the above, $i, j$ denote the mass indices, $L$ is the baseline distance, and $U_{\alpha i}$, etc. are the elements of the PontecorvoMaki-Nakagawa-Sakata (PMNS) mixing matrix [48] such that

$$
\left|\nu_{\alpha}\right\rangle=\sum_{i} U_{\alpha i}\left|\nu_{i}\right\rangle
$$

with $\left|\nu_{i}\right\rangle$ being the $i$ th mass eigenstate. The oscillation length $\lambda_{i j}$ is given by

$$
\lambda_{i j}=2.47 \mathrm{~km}\left(\frac{E}{\mathrm{GeV}}\right)\left(\frac{\mathrm{eV}^{2}}{\Delta m_{i j}^{2}}\right),
$$

where $E$ is the neutrino energy and $\Delta m_{i j}^{2}$ is the mass-squared difference of the $i$ th and $j$ th neutrino mass eigenstates. The baseline distance $L$ of UHE neutrinos is generally astronomical in scale. With $\Delta m^{2} L / E \gg 1$ for UHE neutrinos from distant GRBs or active galactic nuclei, the oscillatory part in the probability equation is averaged to $\frac{1}{2}$. Thus,

$$
\left\langle\sin ^{2}\left(\frac{\pi L}{\lambda_{i j}}\right)\right\rangle=\frac{1}{2}
$$

The probability equation (1) is then reduced to

$$
\begin{aligned}
P_{\nu_{\alpha} \rightarrow \nu_{\beta}} & =\delta_{\alpha \beta}-2 \sum_{j>i} U_{\alpha i} U_{\beta i} U_{\alpha j} U_{\beta j} \\
& =\delta_{\alpha \beta}-\sum_{i} U_{\alpha i} U_{\beta i}\left[\sum_{j \neq i} U_{\alpha j} U_{\beta j}\right] \\
& =\sum_{j}\left|U_{\alpha j}\right|^{2}\left|U_{\beta j}\right|^{2}
\end{aligned}
$$

where we use the unitarity condition

$$
\sum_{i} U_{\alpha i} U_{\beta i}=\delta_{\alpha \beta}
$$

For the four-flavor scenario, where a fourth sterile neutrino $\nu_{s}$ is considered along with the usual three flavors $\nu_{e}, \nu_{\mu}$, and $\nu_{\tau}$, the neutrino flavor eigenstates and mass eigenstates are related by

$$
\left(\begin{array}{c}
\nu_{e} \\
\nu_{\mu} \\
\nu_{\tau} \\
\nu_{s}
\end{array}\right)=\left(\begin{array}{cccc}
\tilde{U}_{e 1} & \tilde{U}_{e 2} & \tilde{U}_{e 3} & \tilde{U}_{e 4} \\
\tilde{U}_{\mu 1} & \tilde{U}_{\mu 2} & \tilde{U}_{\mu 3} & \tilde{U}_{\mu 4} \\
\tilde{U}_{\tau 1} & \tilde{U}_{\tau 2} & \tilde{U}_{\tau 3} & \tilde{U}_{\tau 4} \\
\tilde{U}_{s 1} & \tilde{U}_{s 2} & \tilde{U}_{s 3} & \tilde{U}_{s 4}
\end{array}\right)\left(\begin{array}{c}
\nu_{1} \\
\nu_{1} \\
\nu_{3} \\
\nu_{4}
\end{array}\right),
$$

where $\tilde{U}_{\alpha i}$, etc. [with $i$ being the mass index $(i=1,2,3,4)$ and $\alpha$ being the flavor index $(\alpha=e, \mu, \tau, s)]$ are the elements of the PMNS mixing matrix for the four-flavor case, which can be generated by successive rotations $(R)$ (in terms of six mixing angles $\left.\theta_{14}, \theta_{24}, \theta_{34}, \theta_{13}, \theta_{12}, \theta_{23}\right)$ [49] as

$$
\tilde{U}=R_{34}\left(\theta_{34}\right) R_{24}\left(\theta_{24}\right) R_{14}\left(\theta_{14}\right) R_{23}\left(\theta_{23}\right) R_{13}\left(\theta_{13}\right) R_{12}\left(\theta_{12}\right),
$$

where we consider no $C P$ violation $^{1}$ in the neutrino sector and hence the $C P$ phases are absent. Considering the present four-flavor scenario to be the minimal extension of the three-flavor case by a sterile neutrino, the matrix $\tilde{U}$ can be written as

\footnotetext{
${ }^{1}$ Although evidence of $C P$ violation in the lepton sector is yet to be established, an analysis of T2K data provides a best-fit value of $\delta=-\pi / 2$ at only $2 \sigma$ C.L. Hence, we have neglected $C P$ violation in our work.
} 


$$
\begin{aligned}
\tilde{U}_{(4 \times 4)}= & \left(\begin{array}{cccc}
c_{14} & 0 & 0 & s_{14} \\
-s_{14} s_{24} & c_{24} & 0 & c_{14} s_{24} \\
-c_{24} s_{14} s_{34} & -s_{24} s_{34} & c_{34} & c_{14} c_{24} s_{34} \\
-c_{24} s_{14} c_{34} & -s_{24} c_{34} & -s_{34} & c_{14} c_{24} c_{34}
\end{array}\right) \times\left(\begin{array}{cccc}
\mathcal{U}_{e 1} & \mathcal{U}_{e 2} & \mathcal{U}_{e 3} & 0 \\
\mathcal{U}_{\mu 1} & \mathcal{U}_{\mu 2} & \mathcal{U}_{\mu 3} & 0 \\
\mathcal{U}_{\tau 1} & \mathcal{U}_{\tau 2} & \mathcal{U}_{\tau 3} & 0 \\
0 & 0 & 0 & 1
\end{array}\right) \\
= & \left(\begin{array}{cccc}
c_{14} \mathcal{U}_{e 1} & c_{14} \mathcal{U}_{e 2} & c_{14} \mathcal{U}_{e 3} & s_{14} \\
-s_{14} s_{24} \mathcal{U}_{e 1}+c_{24} \mathcal{U}_{\mu 1} & -s_{14} s_{24} \mathcal{U}_{e 2}+c_{24} \mathcal{U}_{\mu 2} & -s_{14} s_{24} \mathcal{U}_{e 3}+c_{24} \mathcal{U}_{\mu 3} & c_{14} s_{24} \\
-c_{24} s_{14} s_{34} \mathcal{U}_{e 1} & -c_{24} s_{14} s_{34} \mathcal{U}_{e 2} & -c_{24} s_{14} s_{34} \mathcal{U}_{e 3} & \\
-s 24 s 34 \mathcal{U}_{\mu 1} & -s 24 s 34 \mathcal{U}_{\mu 2} & -s 24 s 34 \mathcal{U}_{\mu 3} & c_{14} c_{24} s_{34} \\
+c_{34} \mathcal{U}_{\tau 1} & +c_{34} \mathcal{U}_{\tau 2} & +c_{34} \mathcal{U}_{\tau 3} & \\
-c_{24} c_{34} s_{14} \mathcal{U}_{e 1} & -c_{24} c_{34} s_{14} \mathcal{U}_{e 2} & -c_{24} c_{34} s_{14} \mathcal{U}_{e 3} & \\
-s_{24} c_{34} \mathcal{U}_{\mu 1} & -s_{24} c_{34} \mathcal{U}_{\mu 2} & -s_{24} c_{34} \mathcal{U}_{\mu 3} & c_{14} c_{24} c_{34} \\
-s_{34} \mathcal{U}_{\tau 1} & -s_{34} \mathcal{U}_{\tau 2} & -s_{34} \mathcal{U}_{\tau 3} &
\end{array}\right),
\end{aligned}
$$

where $\mathcal{U}_{\alpha i}$ are the elements of the three-flavor neutrino mixing matrix

$$
\mathcal{U}_{(3 \times 3)}=\left(\begin{array}{lll}
\mathcal{U}_{e 1} & \mathcal{U}_{e 2} & \mathcal{U}_{e 3} \\
\mathcal{U}_{\mu 1} & \mathcal{U}_{\mu 2} & \mathcal{U}_{\mu 3} \\
\mathcal{U}_{\tau 1} & \mathcal{U}_{\tau 2} & \mathcal{U}_{\tau 3}
\end{array}\right)
$$

The matrix $\mathcal{U}_{(3 \times 3)}$ can be expressed as the successive rotations

$$
\mathcal{U}_{(3 \times 3)}=R_{23} R_{13} R_{12},
$$

where

$$
R_{12}=\left(\begin{array}{ccc}
c_{12} & s_{12} & 0 \\
-s_{12} & c_{12} & 0 \\
0 & 0 & 1
\end{array}\right), \quad R_{13}=\left(\begin{array}{ccc}
c_{13} & 0 & s_{13} \\
0 & 1 & 0 \\
-s_{13} & 0 & c_{13}
\end{array}\right), \quad R_{23}=\left(\begin{array}{ccc}
1 & 0 & 0 \\
0 & c_{23} & s_{23} \\
0 & -s_{23} & c_{23}
\end{array}\right)
$$

Therefore,

$$
\mathcal{U}_{(3 \times 3)}=\left(\begin{array}{ccc}
c_{12} c_{13} & s_{12} s_{13} & s_{13} \\
-s_{12} c_{23}-c_{12} s_{23} s_{13} & c_{12} c_{23}-s_{12} s_{23} s_{13} & s_{23} c_{13} \\
s_{12} s_{23}-c_{12} c_{23} s_{13} & -c_{12} s_{23}-s_{12} c_{23} s_{13} & c_{23} c_{13}
\end{array}\right) \text {. }
$$

Following Eq. (5), the oscillation probability $P_{\nu_{\alpha} \rightarrow \nu_{\beta}}^{4}$ (where $\alpha, \beta$ denote the flavor indices) for the four-flavor case can now be represented as [50]

$$
P_{\nu_{\alpha} \rightarrow \nu_{\beta}}^{4} \equiv\left(\begin{array}{cccc}
P_{e e} & P_{e \mu} & P_{e \tau} & P_{e s} \\
P_{\mu e} & P_{\mu \mu} & P_{\mu \tau} & P_{\mu s} \\
P_{\tau e} & P_{\tau \mu} & P_{\tau \tau} & P_{\tau s} \\
P_{s e} & P_{s \mu} & P_{s \tau} & P_{s s}
\end{array}\right) \equiv X X^{T}
$$

with 


$$
X=\left(\begin{array}{cccc}
\left|\tilde{U}_{e 1}\right|^{2} & \left|\tilde{U}_{e 2}\right|^{2} & \left|\tilde{U}_{e 3}\right|^{2} & \left|\tilde{U}_{e 4}\right|^{2} \\
\left|\tilde{U}_{\mu 1}\right|^{2} & \left|\tilde{U}_{\mu 2}\right|^{2} & \left|\tilde{U}_{\mu 3}\right|^{2} & \left|\tilde{U}_{\mu 4}\right|^{2} \\
\left|\tilde{U}_{\tau 1}\right|^{2} & \left|\tilde{U}_{\tau 2}\right|^{2} & \left|\tilde{U}_{\tau 3}\right|^{2} & \left|\tilde{U}_{\tau 4}\right|^{2} \\
\left|\tilde{U}_{s 1}\right|^{2} & \left|\tilde{U}_{s 2}\right|^{2} & \left|\tilde{U}_{s 3}\right|^{2} & \left|\tilde{U}_{s 4}\right|^{2}
\end{array}\right) .
$$

Similarly, for the three-flavor scenario the probability $P_{\nu_{\alpha} \rightarrow \nu_{\beta}}^{3}$ takes the form

$$
P_{\nu_{\alpha} \rightarrow \nu_{\beta}}^{3}=A A^{T},
$$

where

$$
A=\left(\begin{array}{ccc}
\left|\mathcal{U}_{e 1}\right|^{2} & \left|\mathcal{U}_{e 2}\right|^{2} & \left|\mathcal{U}_{e 3}\right|^{2} \\
\left|\mathcal{U}_{\mu 1}\right|^{2} & \left|\mathcal{U}_{\mu 2}\right|^{2} & \left|\mathcal{U}_{\mu 3}\right|^{2} \\
\left|\mathcal{U}_{\tau 1}\right|^{2} & \left|\mathcal{U}_{\tau 2}\right|^{2} & \left|\mathcal{U}_{\tau 3}\right|^{2}
\end{array}\right)
$$

\section{B. UHE neutrino fluxes from GRBs}

Neutrinos and antineutrinos from GRBs are expected to be produced with the flavor ratio

$$
\nu_{e}: \nu_{\mu}: \nu_{\tau}=1: 2: 0
$$

The isotropic flux [51,52] for $\nu_{\mu}$ and $\bar{\nu}_{\mu}$ estimated by summing over all of the sources is given as [53]

$\mathcal{F}\left(E_{\nu}\right)=\frac{d N_{\nu_{\mu}+\bar{\nu}_{\mu}}}{d E_{\nu}}=\mathcal{N}\left(\frac{E_{\nu}}{1 \mathrm{GeV}}\right)^{-n} \mathrm{~cm}^{-2} \mathrm{~s}^{-1} \mathrm{sr}^{-1} \mathrm{GeV}^{-1}$.

In the above,

$$
\begin{aligned}
& \mathcal{N}=4.0 \times 10^{-13}, \quad n=1 \quad \text { for } E_{\nu}<10^{5} \mathrm{GeV}, \\
& \mathcal{N}=4.0 \times 10^{-8}, \quad n=2 \text { for } E_{\nu}>10^{5} \mathrm{GeV}
\end{aligned}
$$

Therefore the fluxes of the corresponding flavors (which are the same for both neutrinos and antineutrinos since no $C P$ violation is considered in the neutrino sector) can be expressed as

$$
\begin{aligned}
& \frac{d N_{\nu_{\mu}}}{d E_{\nu}}=\phi_{\nu_{\mu}}=\frac{d N_{\bar{\nu}_{\mu}}}{d E_{\nu}}=\phi_{\bar{\nu}_{\mu}}=0.5 \mathcal{F}\left(E_{\nu}\right), \\
& \frac{d N_{\nu_{e}}}{d E_{\nu}}=\phi_{\nu_{e}}=\frac{d N_{\bar{\nu}_{e}}}{d E_{\nu}}=\phi_{\bar{\nu}_{e}}=0.25 \mathcal{F}\left(E_{\nu}\right) .
\end{aligned}
$$

These neutrinos suffer flavor oscillations as they reach the terrestrial detector due to the astronomical baseline length. Thus, in the process the $\nu_{\mu}$ can oscillate to $\nu_{\tau}$ and/or to other flavors upon reaching the Earth. The flux of neutrino flavors for the four- and three-flavor cases upon reaching the Earth will be, respectively,

$$
\begin{aligned}
& F_{\nu_{e}}^{4}=P_{\nu_{e} \rightarrow \nu_{e}}^{4} \phi_{\nu_{e}}+P_{\nu_{\mu} \rightarrow \nu_{e}}^{4} \phi_{\nu_{\mu}}, \\
& F_{\nu_{\mu}}^{4}=P_{\nu_{\mu} \rightarrow \nu_{\mu}}^{4} \phi_{\nu_{\mu}}+P_{\nu_{e} \rightarrow \nu_{\mu}}^{4} \phi_{\nu_{e}}, \\
& F_{\nu_{\tau}}^{4}=P_{\nu_{e} \rightarrow \nu_{\tau}}^{4} \phi_{\nu_{e}}+P_{\nu_{\mu} \rightarrow \nu_{\tau}}^{4} \phi_{\nu_{\mu}}, \\
& F_{\nu_{s}}^{4}=P_{\nu_{e} \rightarrow \nu_{s}}^{4} \phi_{\nu_{e}}+P_{\nu_{\mu} \rightarrow \nu_{s}}^{4} \phi_{\nu_{\mu}},
\end{aligned}
$$

and

$$
\begin{aligned}
& F_{\nu_{e}}^{3}=P_{\nu_{e} \rightarrow \nu_{e}}^{3} \phi_{\nu_{e}}+P_{\nu_{\mu} \rightarrow \nu_{e}}^{3} \phi_{\nu_{\mu}}, \\
& F_{\nu_{\mu}}^{3}=P_{\nu_{\mu} \rightarrow \nu_{\mu}}^{3} \phi_{\nu_{\mu}}+P_{\nu_{e} \rightarrow \nu_{\mu}}^{3} \phi_{\nu_{e}}, \\
& F_{\nu_{\tau}}^{3}=P_{\nu_{e} \rightarrow \nu_{\tau}}^{3} \phi_{\nu_{e}}+P_{\nu_{\mu} \rightarrow \nu_{\tau}}^{3} \phi_{\nu_{\mu}} .
\end{aligned}
$$

In the above, $F_{\nu_{\alpha}}^{4}\left(F_{\nu_{\alpha}}^{3}\right)$ is the flux for the species $\nu_{\alpha}$, with $\alpha$ being the flavor index, and $P_{\nu_{\alpha}}^{4}\left(P^{3}{ }_{\nu_{\alpha}}\right)$ is the corresponding oscillation probability for the four- (three-)flavor scenario.

The distant cosmic neutrino flux (21) can be expressed as the product of $P_{(4 \times 4)}\left(=X X^{T}\right)$ and the intrinsic flux $\phi_{\nu_{\alpha}}(\alpha=e, \nu, \tau, s)$ in matrix form,

$$
\left(\begin{array}{c}
F_{\nu_{e}}^{4} \\
F_{\nu_{\mu}}^{4} \\
F_{\nu_{\tau}}^{4} \\
F_{\nu_{s}}^{4}
\end{array}\right)=X X^{T} \times\left(\begin{array}{c}
\phi_{\nu_{e}} \\
\phi_{\nu_{\mu}} \\
\phi_{\nu_{\tau}} \\
\phi_{\nu_{s}}
\end{array}\right)
$$

We assume the standard ratio for the intrinsic neutrino flux, i.e.,

$$
\phi_{\nu_{e}}: \phi_{\nu_{\mu}}: \phi_{\nu_{\tau}}: \phi_{\nu_{s}}=1: 2: 0: 0
$$

Now by using the above assumption and Eqs. (16), (23) can be rewritten as

$$
\begin{aligned}
\left(\begin{array}{c}
F_{\nu_{e}}^{4} \\
F_{\nu_{\mu}}^{4} \\
F_{\nu_{\tau}}^{4} \\
F_{\nu_{s}}^{4}
\end{array}\right) & =\left(\begin{array}{llll}
\left|\tilde{U}_{e 1}\right|^{2} & \left|\tilde{U}_{e 2}\right|^{2} & \left|\tilde{U}_{e 3}\right|^{2} & \left|\tilde{U}_{e 4}\right|^{2} \\
\left|\tilde{U}_{\mu 1}\right|^{2} & \left|\tilde{U}_{\mu 2}\right|^{2} & \left|\tilde{U}_{\mu 3}\right|^{2} & \left|\tilde{U}_{\mu 4}\right|^{2} \\
\left|\tilde{U}_{\tau 1}\right|^{2} & \left|\tilde{U}_{\tau 2}\right|^{2} & \left|\tilde{U}_{\tau 3}\right|^{2} & \left|\tilde{U}_{\tau 4}\right|^{2} \\
\left|\tilde{U}_{s 1}\right|^{2} & \left|\tilde{U}_{s 2}\right|^{2} & \left|\tilde{U}_{s 3}\right|^{2} & \left|\tilde{U}_{s 4}\right|^{2}
\end{array}\right) \\
& \times\left(\begin{array}{llll}
\left|\tilde{U}_{e 1}\right|^{2} & \left|\tilde{U}_{\mu 1}\right|^{2} & \left|\tilde{U}_{\tau 1}\right|^{2} & \left|\tilde{U}_{s 1}\right|^{2} \\
\left|\tilde{U}_{e 2}\right|^{2} & \left|\tilde{U}_{\mu 2}\right|^{2} & \left|\tilde{U}_{\tau 2}\right|^{2} & \left|\tilde{U}_{s 2}\right|^{2} \\
\left|\tilde{U}_{e 3}\right|^{2} & \left|\tilde{U}_{\mu 3}\right|^{2} & \left|\tilde{U}_{\tau 3}\right|^{2} & \left|\tilde{U}_{s 3}\right|^{2} \\
\left|\tilde{U}_{e 4}\right|^{2} & \left|\tilde{U}_{\mu 4}\right|^{2} & \left|\tilde{U}_{\tau 4}\right|^{2} & \left|\tilde{U}_{s 4}\right|^{2}
\end{array}\right)\left(\begin{array}{l}
1 \\
2 \\
0 \\
0
\end{array}\right) \phi_{\nu_{e}} .
\end{aligned}
$$

From Eq. (24), it then follows that 


$$
\begin{aligned}
F_{\nu_{e}}^{4}= & {\left[\left|\tilde{U}_{e 1}\right|^{2}\left(1+\left|\tilde{U}_{\mu 1}\right|^{2}-\left|\tilde{U}_{\tau 1}\right|^{2}-\left|\tilde{U}_{s 1}\right|^{2}\right)+\left|\tilde{U}_{e 2}\right|^{2}\left(1+\left|\tilde{U}_{\mu 2}\right|^{2}-\left|\tilde{U}_{\tau 2}\right|^{2}-\left|\tilde{U}_{s 2}\right|^{2}\right)\right.} \\
& \left.+\left|\tilde{U}_{e 3}\right|^{2}\left(1+\left|\tilde{U}_{\mu 3}\right|^{2}-\left|\tilde{U}_{\tau 3}\right|^{2}-\left|\tilde{U}_{s 3}\right|^{2}\right)+\left|\tilde{U}_{e 4}\right|^{2}\left(1+\left|\tilde{U}_{\mu 4}\right|^{2}-\left|\tilde{U}_{\tau 4}\right|^{2}-\left|\tilde{U}_{s 4}\right|^{2}\right)\right] \phi_{\nu_{e}}, \\
F_{\nu_{\mu}}^{4}= & {\left[\left|\tilde{U}_{\mu 1}\right|^{2}\left(1+\left|\tilde{U}_{\mu 1}\right|^{2}-\left|\tilde{U}_{\tau 1}\right|^{2}-\left|\tilde{U}_{s 1}\right|^{2}\right)+\left|\tilde{U}_{\mu 2}\right|^{2}\left(1+\left|\tilde{U}_{\mu 2}\right|^{2}-\left|\tilde{U}_{\tau 2}\right|^{2}-\left|\tilde{U}_{s 2}\right|^{2}\right)\right.} \\
& \left.+\left|\tilde{U}_{\mu 3}\right|^{2}\left(1+\left|\tilde{U}_{\mu 3}\right|^{2}-\left|\tilde{U}_{\tau 3}\right|^{2}-\left|\tilde{U}_{s 3}\right|^{2}\right)+\left|\tilde{U}_{\mu 4}\right|^{2}\left(1+\left|\tilde{U}_{\mu 4}\right|^{2}-\left|\tilde{U}_{\tau 4}\right|^{2}-\left|\tilde{U}_{s 4}\right|^{2}\right)\right] \phi_{\nu_{e}}, \\
F_{\nu_{\tau}}^{4}= & {\left[\left|\tilde{U}_{\tau 1}\right|^{2}\left(1+\left|\tilde{U}_{\mu 1}\right|^{2}-\left|\tilde{U}_{\tau 1}\right|^{2}-\left|\tilde{U}_{s 1}\right|^{2}\right)+\left|\tilde{U}_{\tau 2}\right|^{2}\left(1+\left|\tilde{U}_{\mu 2}\right|^{2}-\left|\tilde{U}_{\tau 2}\right|^{2}-\left|\tilde{U}_{s 2}\right|^{2}\right)\right.} \\
& \left.+\left|\tilde{U}_{\tau 3}\right|^{2}\left(1+\left|\tilde{U}_{\mu 3}\right|^{2}-\left|\tilde{U}_{\tau 3}\right|^{2}-\left|\tilde{U}_{s 3}\right|^{2}\right)+\left|\tilde{U}_{\tau 4}\right|^{2}\left(1+\left|\tilde{U}_{\mu 4}\right|^{2}-\left|\tilde{U}_{\tau 4}\right|^{2}-\left|\tilde{U}_{s 4}\right|^{2}\right)\right] \phi_{\nu_{e}}, \\
F_{\nu_{s}}^{4}= & {\left[\left|\tilde{U}_{s 1}\right|^{2}\left(1+\left|\tilde{U}_{\mu 1}\right|^{2}-\left|\tilde{U}_{\tau 1}\right|^{2}-\left|\tilde{U}_{s 1}\right|^{2}\right)+\left|\tilde{U}_{s 2}\right|^{2}\left(1+\left|\tilde{U}_{\mu 2}\right|^{2}-\left|\tilde{U}_{\tau 2}\right|^{2}-\left|\tilde{U}_{s 2}\right|^{2}\right)\right.} \\
& \left.+\left|\tilde{U}_{s 3}\right|^{2}\left(1+\left|\tilde{U}_{\mu 3}\right|^{2}-\left|\tilde{U}_{\tau 3}\right|^{2}-\left|\tilde{U}_{s 3}\right|^{2}\right)+\left|\tilde{U}_{s 4}\right|^{2}\left(1+\left|\tilde{U}_{\mu 4}\right|^{2}-\left|\tilde{U}_{\tau 4}\right|^{2}-\left|\tilde{U}_{s 4}\right|^{2}\right)\right] \phi_{\nu_{e}}
\end{aligned}
$$

Similarly, for the three-flavor scenario we can write Eq. (22) by using Eqs. (17)-(18) as

$$
\left(\begin{array}{c}
F_{\nu_{e}}^{3} \\
F_{\nu_{\mu}}^{3} \\
F_{\nu_{\tau}}^{3}
\end{array}\right)=\left(\begin{array}{lll}
\left|\mathcal{U}_{e 1}\right|^{2} & \left|\mathcal{U}_{e 2}\right|^{2} & \left|\mathcal{U}_{e 3}\right|^{2} \\
\left|\mathcal{U}_{\mu 1}\right|^{2} & \left|\mathcal{U}_{\mu 2}\right|^{2} & \left|\mathcal{U}_{\mu 3}\right|^{2} \\
\left|\mathcal{U}_{\tau 1}\right|^{2} & \left|\mathcal{U}_{\tau 2}\right|^{2} & \left|\mathcal{U}_{\tau 3}\right|^{2}
\end{array}\right)\left(\begin{array}{ccc}
\left|\mathcal{U}_{e 1}\right|^{2} & \left|\mathcal{U}_{\mu 1}\right|^{2} & \left|\mathcal{U}_{\tau 1}\right|^{2} \\
\left|\mathcal{U}_{e 2}\right|^{2} & \left|\mathcal{U}_{\mu 2}\right|^{2} & \left|\mathcal{U}_{\tau 2}\right|^{2} \\
\left|\mathcal{U}_{e 3}\right|^{2} & \left|\mathcal{U}_{\mu 3}\right|^{2} & \left|\mathcal{U}_{\tau 3}\right|^{2}
\end{array}\right)\left(\begin{array}{l}
1 \\
2 \\
0
\end{array}\right) \phi_{\nu_{e}} .
$$

Finally, Eq. (26) can be written as

$$
\begin{aligned}
& F_{\nu_{e}}^{3}=\left[\left|\mathcal{U}_{e 1}\right|^{2}\left(1+\left|\mathcal{U}_{\mu 1}\right|^{2}-\left|\mathcal{U}_{\tau 1}\right|^{2}\right)+\left|\mathcal{U}_{e 2}\right|^{2}\left(1+\left|\mathcal{U}_{\mu 2}\right|^{2}-\left|\mathcal{U}_{\tau 2}\right|^{2}\right)+\left|\mathcal{U}_{e 3}\right|^{2}\left(1+\left|\mathcal{U}_{\mu 3}\right|^{2}-\left|\mathcal{U}_{\tau 3}\right|^{2}\right)\right] \phi_{\nu_{e}}, \\
& F_{\nu_{\mu}}^{3}=\left[\left|\mathcal{U}_{\mu 1}\right|^{2}\left(1+\left|\mathcal{U}_{\mu 1}\right|^{2}-\left|\mathcal{U}_{\tau 1}\right|^{2}\right)+\left|\mathcal{U}_{\mu 2}\right|^{2}\left(1+\left|\mathcal{U}_{\mu 2}\right|^{2}-\left|\mathcal{U}_{\tau 2}\right|^{2}\right)+\left|\mathcal{U}_{\mu 3}\right|^{2}\left(1+\left|\mathcal{U}_{\mu 3}\right|^{2}-\left|\mathcal{U}_{\tau 3}\right|^{2}\right)\right] \phi_{\nu_{e}}, \\
& F_{\nu_{\tau}}^{3}=\left[\left|\mathcal{U}_{\tau 1}\right|^{2}\left(1+\left|\mathcal{U}_{\mu 1}\right|^{2}-\left|\mathcal{U}_{\tau 1}\right|^{2}\right)+\left|\mathcal{U}_{\tau 2}\right|^{2}\left(1+\left|\mathcal{U}_{\mu 2}\right|^{2}-\left|\mathcal{U}_{\tau 2}\right|^{2}\right)+\left|\mathcal{U}_{\tau 3}\right|^{2}\left(1+\left|\mathcal{U}_{\mu 3}\right|^{2}-\left|\mathcal{U}_{\tau 3}\right|^{2}\right)\right] \phi_{\nu_{e}} .
\end{aligned}
$$

\section{Detection of UHE neutrinos from diffuse GRB sources}

The most promising method of detection is to look for upward-going muons produced by $\nu_{\mu} \mathrm{CC}$ interactions. Such upward-going muons cannot be misidentified as muons produced in the atmosphere. The detection of $\nu_{\mu}$ 's from GRBs can be observed from the tracks of the secondary muons.

The total number of secondary muons that can be observed in a detector of unit area is (following Refs. [54-56])

$$
S=\int_{E_{\mathrm{thr}}}^{E_{\nu_{\max }}} d E_{\nu} \frac{d N_{\nu}}{d E_{\nu}} P_{\text {shadow }}\left(E_{\nu}\right) P_{\mu}\left(E_{\nu}, E_{\mathrm{thr}}\right) .
$$

The phenomenon of Earth shielding can be described by the shadow factor $P_{\text {shadow }}\left(E_{\nu}\right)$, which is defined to be the effective solid angle divided by $2 \pi$ for upward-going muons. This is a function of the energy-dependent neutrino-nucleon interaction length $L_{\text {int }}\left(E_{\nu}\right)$ in the Earth and the column depth $z\left(\theta_{z}\right)$ for the incident neutrino zenith angle $\theta_{z}$. For the case of isotropic fluxes, the attenuation can be represented by this shadow factor, which is given by

$P_{\text {shadow }}\left(E_{\nu}\right)=\frac{1}{2 \pi} \int_{-1}^{0} d \cos \theta_{z} \int d \phi \exp \left[-z\left(\theta_{z}\right) / L_{\text {int }}\left(E_{\nu}\right)\right]$, where the interaction length $L_{\text {int }}\left(E_{\nu}\right)$ is given by

$$
L_{\mathrm{int}}=\frac{1}{\sigma^{\mathrm{tot}}\left(E_{\nu}\right) N_{A}} .
$$

In the above expression, $N_{A}\left(=6.023 \times 10^{23} \mathrm{~mol}^{-1}=6.023 \times\right.$ $\left.10^{23} \mathrm{~cm}^{-3}\right)$ is Avogadro's number and $\sigma^{\text {tot }}\left(=\sigma^{\mathrm{NC}}+\sigma^{\mathrm{CC}}\right)$ is the total (charged-current plus neutral-current) cross section. The column depth $z\left(\theta_{z}\right)$ can be expressed as

$$
z\left(\theta_{z}\right)=\int \rho\left(r\left(\theta_{z}, l\right)\right) d l
$$

In Eq. (31), $\rho\left(r\left(\theta_{z}, l\right)\right)$ represents the density of the Earth. To a good approximation, the Earth may be considered as a spherically symmetric ball consisting of a dense inner and outer core and a lower mantle of medium density. In our work we consider a convenient representation of the matter density profile of the Earth, which is given by the Preliminary reference Earth model (PREM) [57]. The neutrino path length in the Earth is $l$.

The probability $P_{\mu}\left(E_{\nu}, E_{\mathrm{thr}}\right)$ for a muon arriving at the detector with an energy threshold of $E_{\mathrm{thr}}$ is given by

$$
P_{\mu}\left(E_{\nu}, E_{\mathrm{thr}}\right)=N_{A} \sigma^{\mathrm{cc}}\left(E_{\nu}\right)\left\langle R\left(E_{\mu} ; E_{\mathrm{thr}}\right)\right\rangle,
$$


where $\left\langle R\left(E_{\mu} ; E_{\text {thr }}\right)\right\rangle$ is the average range of a muon in the Earth's interior, which can be expressed as

$$
\begin{aligned}
\left\langle R\left(E_{\mu} ; E_{\mathrm{thr}}\right)\right\rangle= & \frac{1}{\sigma_{\mathrm{CC}}} \int_{0}^{\left(1-E_{\mathrm{thr}} / E_{\nu}\right)} d y R\left(E_{\nu}(1-y) ; E_{\mathrm{thr}}\right) \\
& \times \frac{d \sigma_{\mathrm{CC}}\left(E_{\nu}, y\right)}{d y} .
\end{aligned}
$$

In the above equation, the fraction of energy lost by a neutrino with energy $E_{\nu}$ due to the charged-current interactions with a secondary muon of energy $E_{\mu}$ can be represented as $y=\left(E_{\nu}-E_{\mu}\right) / E_{\nu}$. So the range for a muon of energy $E_{\mu}$ is given by

$R\left(E_{\mu} ; E_{\mathrm{thr}}\right)=\int_{E_{\mathrm{thr}}}^{E_{\mu}} \frac{d E_{\mu}}{\left\langle d E_{\mu} / d X\right\rangle} \simeq \frac{1}{\xi} \ln \left(\frac{\alpha+\xi E_{\mu}}{\alpha+\xi E_{\mathrm{thr}}}\right)$.

The energy loss rate of muons with energy $E_{\mu}$ due to ionization and catastrophic losses (like bremsstrahlung, pair production, and hadron production) is expressed as [55]

$$
\left\langle\frac{d E_{\mu}}{d X}\right\rangle=-\alpha-\xi E_{\mu}
$$

The constants $\alpha$ and $\xi$ in Eq. (35) describe the energy losses and the catastrophic losses, respectively, in the Earth's interior. These two constants are computed as

$$
\begin{aligned}
& \alpha=2.033+0.077 \ln \left[E_{\mu}(\mathrm{GeV})\right] \times 10^{3} \mathrm{GeV} \mathrm{cm}^{2} \mathrm{gm}^{-1}, \\
& \xi=2.033+0.077 \ln \left[E_{\mu}(\mathrm{GeV})\right] \times 10^{-6} \mathrm{GeV} \mathrm{cm}^{2} \mathrm{gm}^{-1}
\end{aligned}
$$

for $E_{\mu} \leq 10^{6} \mathrm{GeV}$ [58], and otherwise [59]

$$
\begin{aligned}
\alpha & =2.033 \times 10^{-3} \mathrm{GeV} \mathrm{cm}^{2} \mathrm{gm}^{-1}, \\
\xi & =3.9 \times 10^{-6} \mathrm{GeV} \mathrm{cm}^{2} \mathrm{gm}^{-1} .
\end{aligned}
$$

As mentioned earlier, we also consider muon events from $\tau$ decay $\left[\tau\left(\nu_{\tau}+N \rightarrow \tau+X\right)\right]$ which is produced via the CC interactions of $\nu_{\tau}$ in the Earth.

The muon events from charged-current interactions can be computed by replacing $\frac{d N_{\nu}}{d E_{\nu}}$ in Eq. (28) by $F_{\nu_{\mu}}^{4}$ from Eq. (25) and $F_{\nu_{\mu}}^{3}$ from Eq. (27) for the four-flavor and threeflavor scenarios, respectively.

The only way to consider this process is to assume that after a very short distance this $\tau$ decays back to $\nu_{\tau}$ plus leptons, and the process occurs with a probability of 0.18 $[60,61]$. Using Eqs. (28)-(37), the number of such muon events can be computed.

We consider the shower events from the CC interactions of $\nu+e$ and from the NC interactions of all three active flavors. For the shower case, we consider the whole detector volume $V$ and neglect any specific track events. For the shower case, the event rate is given by
$S_{\mathrm{sh}}=V \int_{E_{\mathrm{thr}}}^{E_{\nu_{\max }}} d E_{\nu} \frac{d N_{\nu}}{d E_{\nu}} P_{\text {shadow }}\left(E_{\nu}\right) \int d y \frac{1}{\sigma^{i}} \frac{d \sigma^{i}}{d y} P_{\text {int }}\left(E_{\nu}, y\right)$.

In the above expression, $\sigma^{i}=\sigma^{\mathrm{CC}}$ for the electromagnetic shower and $\sigma^{i}=\sigma^{\mathrm{NC}}$ when $\nu_{e}-\nu_{\mu} \mathrm{NC}$ interactions are considered. The probability that a shower is produced by the neutrino interactions is given by

$$
P_{\mathrm{int}}=\rho N_{A} \sigma^{i} L,
$$

where $\rho$ is the matter density and $L$ is the length of the detector. According to the case of shower events, $\frac{d N_{\nu}}{d E_{\nu}}$ in Eq. (38) is replaced by $F_{\nu_{e}}^{4}, F_{\nu_{\mu}}^{4}, F_{\nu_{\tau}}^{4}$ from Eq. (25) and $F_{\nu_{e}}^{3}$, $F_{\nu_{\mu}}^{3}, F_{\nu_{\tau}}^{3}$ from Eq. (27) for the four-flavor and three-flavor scenarios, respectively.

\section{Detection of neutrinos from a single GRB}

In this subsection we consider muon events from neutrinos that come from a single GRB. We follow a similar approach as in Sec. II C (diffuse GRB case) for this purpose. Beside the fact that the expression for the flux for a single GRB is different than that for the case for diffuse GRBs, the zenith angle $\theta_{z}$ [used in Eq. (29)] is now fixed for a particular GRB. Thus, the expression for $P_{\text {shadow }}$ is now modified as

$$
P_{\text {shadow }}=\exp \left[-z\left(\theta_{z}\right) / L_{\text {int }}\left(E_{\nu}\right)\right] .
$$

The Earth's density should also be accordingly computed for a fixed $\theta_{z}$.

For the case of isotropic emission from the source, the secondary neutrino flux $\frac{d N_{\nu 0}}{d E_{\nu \mathrm{obs}}}$ (the total number of secondary neutrinos emitted from a single GRB at redshift $z^{\prime}$ per unit observed neutrino energy $E_{\nu \text { obs }}$ that are incident on the Earth) is given by

$$
\frac{d N_{\nu 0}}{d E_{\nu \mathrm{obs}}}=\frac{d N_{\nu}}{d E_{\nu}} \frac{1}{4 \pi r^{2}\left(z^{\prime}\right)}\left(1+z^{\prime}\right),
$$

where the comoving radial coordinate distance $r\left(z^{\prime}\right)$ of the source is expressed as

$$
r\left(z^{\prime}\right)=\frac{c}{H_{0}} \int_{0}^{z^{\prime}} \frac{d z^{\prime \prime}}{\sqrt{\Omega_{\Lambda}+\Omega_{m}\left(1+z^{\prime \prime}\right)^{3}}} .
$$

In a spatially flat universe $\Omega_{\Lambda}+\Omega_{m}=1$, where $\Omega_{\Lambda}$ is the energy component of the critical energy density of the universe and $\Omega_{m}$ is the contribution of the matter density to the energy density of the universe in units of the critical energy density. The speed of light is denoted as $c$ and $H_{0}$ is the Hubble constant. The values of the constants adopted in our calculation are $\Omega_{\Lambda}=0.684, \Omega_{m}=0.316$, and $H_{0}=$ $67.8 \mathrm{~km} \mathrm{~s}^{-1} \mathrm{Mpc}^{-1}$. 
The neutrino spectrum $\frac{d N_{\nu}}{d E_{\nu}}$ in Eq. (41) is expressed as

$$
\frac{d N_{\nu}}{d E_{\nu}}=N \times \min \left(1, \frac{E_{\nu}}{E_{\nu}^{\mathrm{br}}}\right) \frac{1}{E_{\nu}^{2}} .
$$

In the above, $N$ is the normalization constant and $E_{\nu}^{\mathrm{br}}$ is the neutrino spectrum break energy. The latter $\left(E_{\nu}^{\mathrm{br}}\right)$ is a function of the Lorentz factor of the GRB $(\Gamma)$ and the photon spectral break energy $\left(E_{\gamma, \mathrm{MeV}}^{\mathrm{br}}\right)$, and is given by

$$
E_{\nu}^{\mathrm{br}} \equiv 10^{6} \frac{\Gamma_{2.5}^{2}}{E_{\gamma, \mathrm{MeV}}^{\mathrm{br}}} \mathrm{GeV}
$$

where $\Gamma_{2.5}=\Gamma / 10^{2.5}$. The normalization constant $N$ can be written as

$$
N=\frac{E_{\mathrm{GRB}}}{1+\ln \left(E_{\nu \max } / E_{\nu}^{\mathrm{br}}\right)} .
$$

In the above, $E_{\nu \max } E_{\nu \text { min }}$ represent the lower and upper cutoff energies of the neutrino spectrum, respectively. At the time of neutrino emission from a single GRB the total amount of energy released is $E_{\mathrm{GRB}}$, which is $10 \%$ of the total fireball proton energy.

With the neutrino flux from a single GRB computed using Eqs. (43)-(45), the same methodology as in the diffuse case is now followed to obtain the muon and shower yields at a $1 \mathrm{~km}^{2}$ detector such as IceCube.

\section{CALCULATIONS AND RESULTS}

In this section we describe the calculations and results for the neutrino-induced muons and the shower events as estimated for a $1 \mathrm{~km}^{2}$ detector. The UHE neutrinos considered here are a) from a diffuse neutrino flux and b) from a single GRB.

\section{A. Diffuse neutrino flux}

The possible secondary muon and shower yields at a $1 \mathrm{~km}^{2}$ detector such as IceCube for three-flavor and $(3+1)$-flavor UHE neutrinos from distant GRB sources are calculated by using Eqs. (19)-(27) and (28)-(39). We can also calculate the same for both four-flavor and three-flavor UHE neutrinos from a single GRB source by solving Eqs. (19)-(27) and (40)-(45). The density profile of the Earth following the Preliminary reference Earth model from Ref. [57] and $\nu N$ interaction cross sections including charged-current and neutral-current interactions and their sum from Ref. [53] are used to calculate the secondary fluxes. In this work, using the Waxman-Bahcall flux [51,52], the detector threshold energy $E_{\mathrm{thr}}$ is taken to be $E_{\mathrm{thr}}=1 \mathrm{TeV}$. In the present calculations we assume $E_{\nu \max }=10^{11} \mathrm{GeV}$.

For the purpose of our analysis, we consider a ratio $R$ between the muon and the shower events, which is defined as

$$
R=\frac{T_{\mu}}{T_{\mathrm{sh}}}
$$

where

$$
\begin{aligned}
T_{\mu}= & S\left(\text { for } \nu_{\mu}\right)+S\left(\text { for } \nu_{\tau}\right), \\
T_{\mathrm{sh}}= & S_{\mathrm{sh}}\left(\text { for } \nu_{e} \text { CC interaction }\right) \\
& +S_{\mathrm{sh}}\left(\text { for } \nu_{e} \text { NC interaction }\right) \\
& +S_{\mathrm{sh}}\left(\text { for } \nu_{\mu} \mathrm{NC} \text { interaction }\right) \\
& +S_{\mathrm{sh}}\left(\text { for } \nu_{\tau} \mathrm{NC} \text { interaction }\right),
\end{aligned}
$$

and the quantities $S$ and $S_{\mathrm{sh}}$ are defined in Eqs. (28) and (38), respectively. In the four- and three-flavor scenarios the abovementioned ratio $R$ is denoted as $R_{4}$ and $R_{3}$, respectively.

The motivation for our work is to show how the neutrinoinduced muon and the shower fluxes from distant UHE sources (namely, diffuse GRBs) are affected when a sterile neutrino exists in addition to the three active neutrinos. For this purpose, we compare the ratio $R$ for the $(3+1)$ scenario and the three-active-neutrino scenario. The calculations are made for two different sets of values of the sterile mixing angles $\theta_{14}, \theta_{24}$, and $\theta_{34}$, while the mixing angles for threeneutrino mixing are adopted as the current best-fit values for them. It goes without saying that the other oscillation parameter $\Delta m^{2}$ plays no role in this case, as the oscillation part is averaged out due to the astronomical baseline length. The limits on the four-flavor mixing angles $\left(\theta_{14}, \theta_{24}, \theta_{34}\right)$ are chosen following the four-flavor analyses of different experimental groups, such as MINOS, Daya Bay, Bugey, and NOvA [15,25,33,62-67]. The upper limits on $\theta_{24}$ and $\theta_{34}$ obtained from NOvA [63] are $\theta_{24} \leq 20.8^{\circ}$ and $\theta_{34} \leq 31.2^{\circ}$ assuming $\Delta m_{41}^{2}=0.5 \mathrm{eV}^{2}$. However, according to the MINOS analysis [15], $\theta_{24} \leq 7.3^{\circ}$ and $\theta_{34} \leq 26.6^{\circ}$ for the same value of $\Delta m_{41}^{2}$. The IceCube-DeepCore [68] results considering $\Delta m_{41}^{2}=1 \mathrm{eV}^{2}$ suggest $\theta_{24} \leq 19.4^{\circ}$ and $\theta_{34} \leq 22.8^{\circ}$. Therefore, in the present work we vary both $\theta_{24}$ and $\theta_{34}$ within the limits $2^{\circ} \leq \theta_{24} \leq 20^{\circ}$ and $2^{\circ} \leq \theta_{34} \leq 20^{\circ}$. We also consider limits on $\theta_{14}$ such that $\theta_{14} \leq 4^{\circ}$, consistent with the results from the combined analysis by MINOS, Daya Bay, and Bugey-3 [25] (in the range $0.2 \mathrm{eV}^{2} \leq \Delta m_{41}^{2} \leq 2 \mathrm{eV}^{2}$ ). Using these limits on $\theta_{14}$, $\theta_{24}$, and $\theta_{34}$ we compute the ratios $R_{4}$ and $R_{3}$ for the diffuse fluxes. In Table I, we show the computed values of $R_{4}$ for two representative sets of values for $\theta_{14}, \theta_{24}$, and $\theta_{34}$. The computed value for $R_{3}$, the muon-to-shower ratio for the three-flavor case, is also shown for comparison. From Table I it is obvious that the muon-to-shower ratio increases by a

TABLE I. Comparison of the muon-to-shower ratio for a diffuse GRB neutrino flux (Waxman-Bahcall flux) for the four-flavor $(3+1)$ case compared to the same for the threeflavor case for two sets of active-sterile neutrino mixing angles. See text for details.

\begin{tabular}{lcccc}
\hline \hline$\theta_{14}$ & $\theta_{24}$ & $\theta_{34}$ & $R_{4}$ (in 4f) & $R_{3}$ (in 3f) \\
\hline $3^{\circ}$ & $5^{\circ}$ & $20^{\circ}$ & 9.48 & 1.80 \\
$4^{\circ}$ & $6^{\circ}$ & $15^{\circ}$ & 9.68 & 1.80 \\
\hline \hline
\end{tabular}




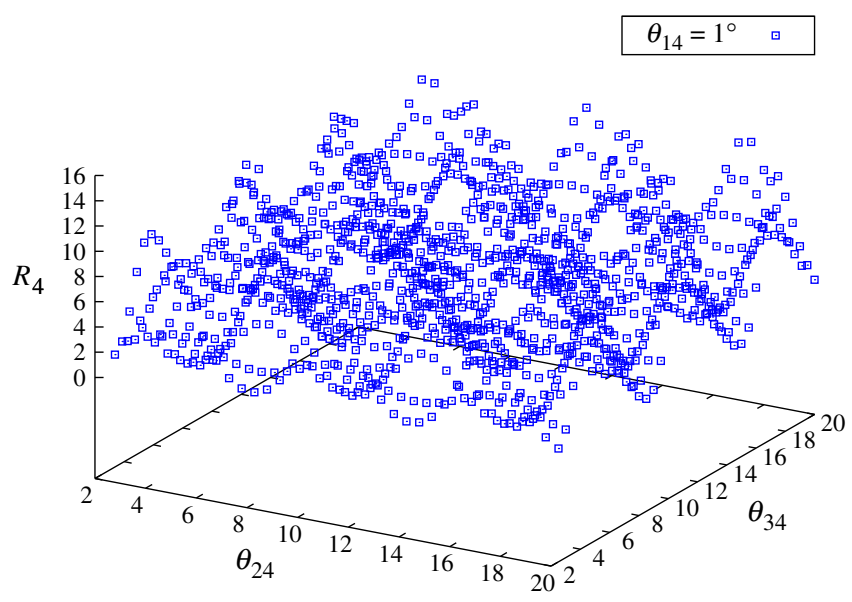

(a)

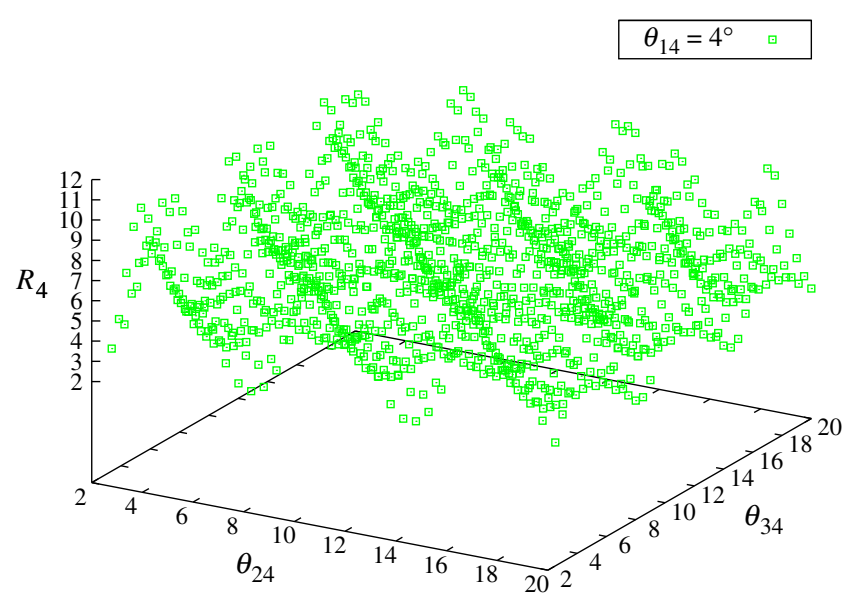

(b)

FIG. 1. Variation of $R_{4}$ with $\theta_{24}$ and $\theta_{34}$ for (a) $\theta_{14}=1^{\circ}$ and (b) $\theta_{14}=4^{\circ}$. See text for details.

considerable amount compared to the ratio for the threeflavor case (for the particular choices shown in Table I, this increase is greater than a factor of 5) if a fourth sterile neutrino is assumed to be present in nature in addition to the three usual active neutrinos.

We also explore how the ratio $R_{4}$ varies with different values of active-sterile mixing angles. In Fig. 1 we show the variations of $R_{4}$ with $\theta_{24}$ and $\theta_{34}$ for two fixed values of $\theta_{14}$, namely, $\theta_{14}=1^{\circ}$ [Fig. 1(a)] and $\theta_{14}=4^{\circ}$ [Fig. 1(b)]. From Fig. 1 it may be noted that the maximum value of the ratio $R_{4}$ is $\sim 6$ times higher than $R_{3}$.

So far, we have discussed our results considering a diffuse flux of UHE neutrinos from several GRBs as obtained from theoretical considerations by Waxman and Bahcall [51,52]. Recently, the IceCube Collaboration published their 6-year data and an analysis based on this data [69]. From the analysis of the high-energy starting events (HESE) data, they calculated a best-fit power law for the neutrino flux as $E^{2} \phi(E)=2.46 \pm 0.8 \times$ $10^{-8}\left(\frac{E}{100 \mathrm{TeV}}\right)^{-0.92} \mathrm{GeV} \mathrm{cm}^{-2} \mathrm{~s}^{-1} \mathrm{sr}^{-1}$. Therefore, for a one-component fit (that is, no broken power law) the neutrino flux $\phi(E) \sim E^{-\gamma}$, with the index $\gamma=2.92$. In fact, their best-fit spectral index is $\gamma=2.92_{-0.29}^{+0.33}$. We have computed $R_{4}, R_{3}$, etc. for this flux as well. Following the astrophysical neutrino results from IceCube [69], one notes that the energy range above $60 \mathrm{TeV}$ is to be considered for such calculations.

The results are shown in Table II. From the table it is seen that the ratio $R_{4}$ from the four-flavor case is $\sim 2$ for the

TABLE II. Same as Table I, but here we consider the diffuse flux of UHE neutrinos obtained from the recent analysis of the IceCube (HESE) data. See text for details.

\begin{tabular}{lcccc}
\hline \hline$\theta_{14}$ & $\theta_{24}$ & $\theta_{34}$ & $R_{4}$ (in 4f) & $R_{3}$ (in 3f) \\
\hline $3^{\circ}$ & $5^{\circ}$ & $20^{\circ}$ & 2.01 & 0.55 \\
$4^{\circ}$ & $6^{\circ}$ & $15^{\circ}$ & 2.04 & 0.55 \\
\hline \hline
\end{tabular}

chosen values of $\theta_{14}, \theta_{24}$, and $\theta_{34}$. Also note that in contrast this track-to-shower ratio is reduced to $\sim 0.55$ for the threeflavor case. Although the ratios are different from those obtained using the Waxman-Bahcall flux, the track-toshower ratio for the four-flavor case is $\sim 3.7$ times that for the three-flavor case. In Figs. 2(a) and 2(b) we show the variations of $R_{4}$ with $\theta_{24}$ and $\theta_{34}$ for $\theta_{14}=1^{\circ}$ and $\theta_{14}=4^{\circ}$, respectively.

\section{B. Single GRB}

We now perform calculations for neutrinos from a single GRB similar to those for the diffuse neutrino flux from several GRBs. A particular GRB occurs at a fixed zenith angle and at a definite redshift with respect to an observer at Earth. We use two sets of active-sterile mixing angles for our calculations, as given in Table I. The active neutrino mixing angles are fixed at their current experimental values. With these sets of parameters, we estimate the neutrinoinduced muons in a $1 \mathrm{~km}^{2}$ detector for the UHE neutrinos from a GRB at different redshifts. The results are obtained using Eqs. (40)-(45) and (14)-(39). The values of the Lorentz factor $\Gamma$ nd photon spectral break energy $E_{\gamma, \mathrm{MeV}}^{\mathrm{br}}$ used to calculate the neutrino flux from a single GRB are chosen as $\Gamma=50.12$ and $E_{\gamma, \mathrm{MeV}}^{\mathrm{br}}=0.794$. These values are adopted from Table I of Ref. [56]. The results are shown in Fig. 3. In Fig. 4 we show the variations of the neutrinoinduced muons with different GRB energies. From both Figs. 3 and 4 it can be observed that the case of four-flavor mixing cannot be distinguished from three-flavor mixing as there is no significant deviation, as observed in the case of the diffuse flux discussed earlier in Sec. III A.

\section{NEUTRINOLESS DOUBLE-BETA DECAY IN THE $3+1$ SCENARIO}

In earlier sections we showed how a four-flavor scenario with three active and one sterile neutrino can affect the 

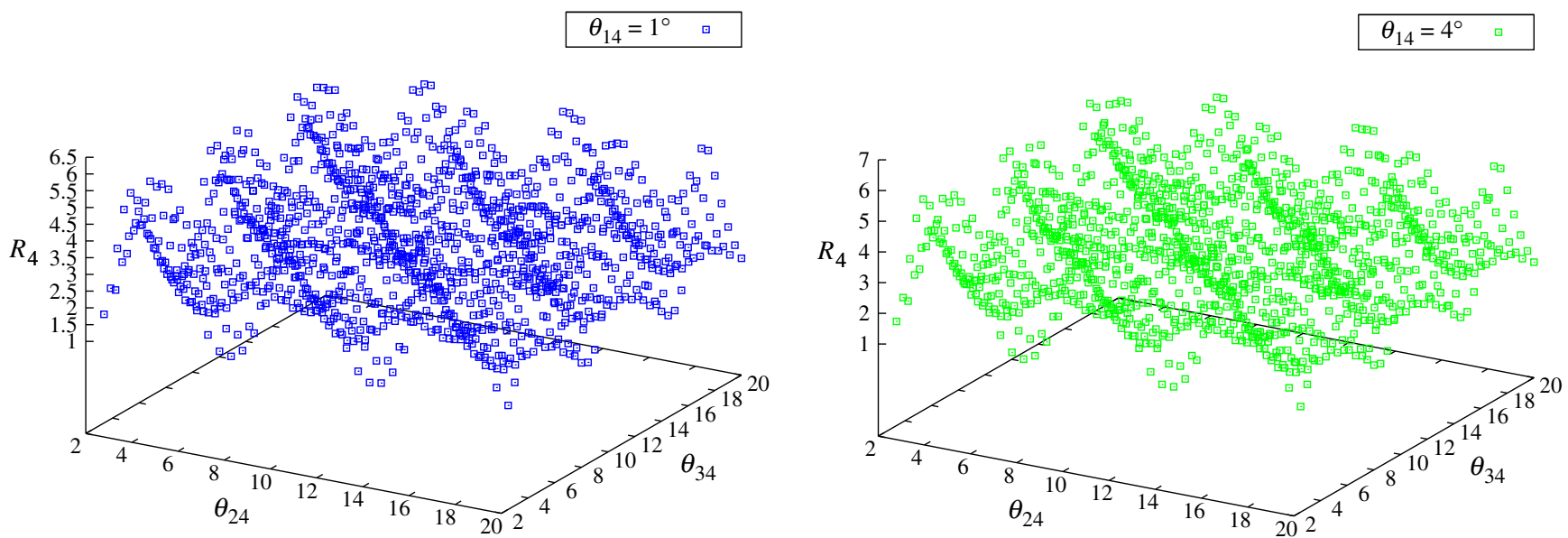

FIG. 2. Variation of $R_{4}$ with $\theta_{24}$ and $\theta_{34}$ for (a) $\theta_{14}=1^{\circ}$ and (b) $\theta_{14}=4^{\circ}$ (the UHE neutrino diffuse flux has been taken from the recent IceCube HESE data). See text for details.

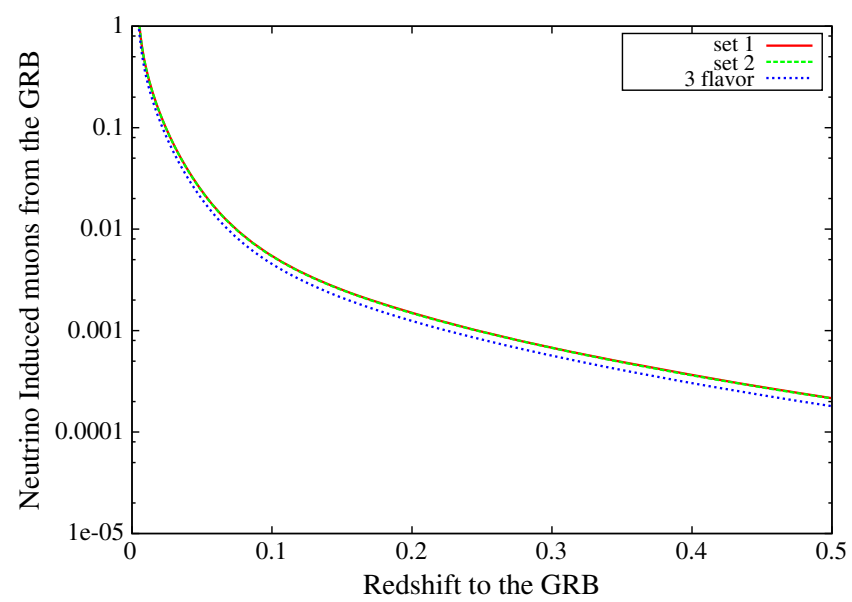

FIG. 3. Variation of the neutrino-induced muons from single GRBs with different redshifts at a fixed zenith angle $\theta_{z}=10^{\circ}$. "set 1" and "set 2" correspond to the two sets of values for activesterile mixing angles given in Table I.

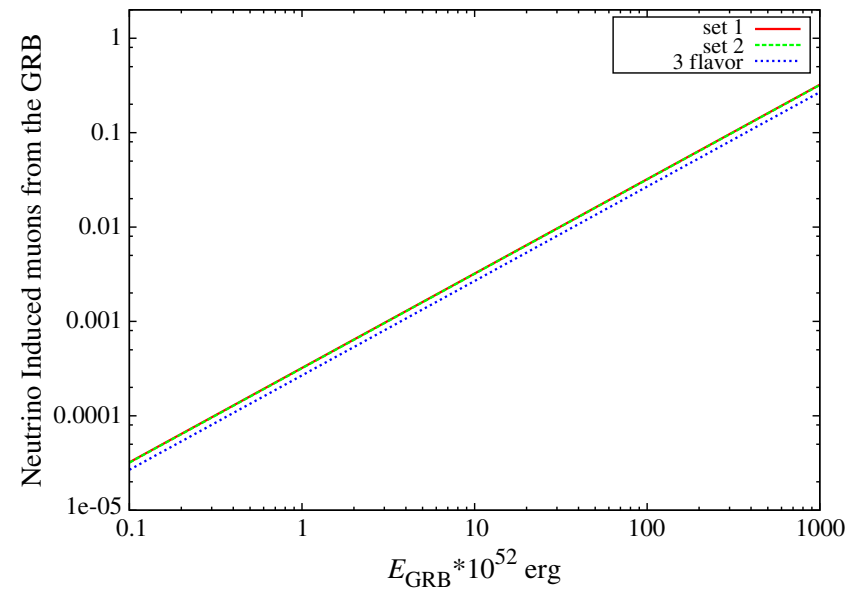

FIG. 4. Variation of the neutrino-induced muons from GRBs with different energies at a fixed zenith angle $\left(\theta_{z}=10^{\circ}\right)$. "set 1 " and "set 2" are as in Fig. 2. neutrino flux for diffuse and single GRB sources when compared with the conventional three-flavor approach. However, these studies do not provide any information about the mass of the sterile neutrino or, more precisely, $\Delta m_{41}^{2}\left(\Delta m_{43}^{2}\right)$ for the normal (inverted) hierarchy of neutrino masses. This is obvious, as the study of GRB fluxes involves large distances and mass-squared oscillations are therefore averaged out. However, the sterile neutrino in the present $3+1$ framework can affect the phenomena of neutrinoless double-beta decay. The effective Majorana mass for observable neutrinoless double-beta decay in the $3+1$ scenario is given as

$$
m_{e e}=\sum_{i=1-4}\left|U_{e i}\right|^{2} m_{i}
$$

where we have neglected the Majorana phases. Equation (48) can be rewritten in terms of the mixing angles,

$$
\begin{aligned}
m_{e e}= & \left|c_{14} c_{12} c_{13}\right|^{2} m_{1}+\left|c_{14} s_{12} c_{13}\right|^{2} m_{2} \\
& +\left|c_{14} s_{13}\right|^{2} m_{3}+\left|s_{14}\right|^{2} m_{4} .
\end{aligned}
$$

We consider that the sterile neutrino with mass $m_{4}$ is heavier than the light active neutrinos. Therefore, the effective Majorana mass in the case of normal ordering of active neutrinos is given as

$$
\begin{aligned}
m_{e e}= & \left|c_{14} c_{12} c_{13}\right|^{2} m_{1}+\left|c_{14} s_{12} c_{13}\right|^{2} \sqrt{m_{1}^{2}+\Delta m_{21}^{2}} \\
& +\left|c_{14} s_{13}\right|^{2} \sqrt{m_{1}^{2}+\Delta m_{31}^{2}} \\
& +\left|s_{14}\right|^{2} \sqrt{m_{1}^{2}+\Delta m_{41}^{2}} .
\end{aligned}
$$

Similarly for the case of the inverted hierarchy of active neutrinos, the expression in Eq. (49) can be rewritten as 


$$
\begin{aligned}
m_{e e}= & \left|c_{14} c_{12} c_{13}\right|^{2} \sqrt{m_{3}^{2}+\Delta m_{23}^{2}-\Delta m_{21}^{2}} \\
& +\left|c_{14} s_{12} c_{13}\right|^{2} \sqrt{m_{3}^{2}+\Delta m_{23}^{2}}+\left|c_{14} s_{13}\right|^{2} m_{3} \\
& +\left|s_{14}\right|^{2} \sqrt{m_{3}^{2}+\Delta m_{43}^{2}} .
\end{aligned}
$$

Hence, for the normal (inverted) hierarchy, $m_{1}\left(m_{3}\right)$ is the lightest neutrino mass, which we will denote as $m_{0}$ for simplicity. From Eqs. (50)-(51), it can be easily observed that the effective Majorana mass $m_{e e}$ depends on new physics involving the sterile neutrino mixing angle $\theta_{14}$ and mass-squared difference $\Delta m_{41}^{2}$ (or, equivalently, $\Delta m_{43}^{2}$ ). In the present work, we investigate the effects of these parameters on the effective Majorana mass for neutrinoless double-beta decay. Since $m_{3}$ is the lightest neutrino in the case of inverted hierarchy, $\Delta m_{43}^{2}=m_{4}^{2}-m_{0}^{2}$ is equivalent to $\Delta m_{41}^{2}=m_{4}^{2}-m_{0}^{2}$ appearing in Eq. (50) for the normal hierarchy. In Fig. 5, we plot the variation of the effective Majorana mass with the lightest neutrino mass $m_{0}$ varied within the range $10^{-3} \mathrm{eV} \leq m_{0} \leq 1 \mathrm{eV}$ for both the normal and inverted hierarchies of neutrino masses using best-fit values of the active neutrino mixing angles $\theta_{12}$ and $\theta_{13}$. The shaded region shown in gray (black) in Fig. 5 corresponds to the normal (inverted) hierarchy of active neutrinos. We consider a conservative limit on the mixing angle between $0^{\circ} \leq \theta_{14} \leq 4^{\circ}$ and the range of $\Delta m_{41}^{2}$ is $0.2-2 \mathrm{eV}^{2}$, consistent with the exclusion limits on $\theta_{14}$ obtained from the combined results of MINOS, Daya Bay, and Bugey-3 (see Ref. [25] and references therein) for the normal hierarchy. We assume the same range of $\theta_{14}$ and $\Delta m_{43}^{2}$ for the case of the inverted hierarchy of neutrino mixing. From Fig. 5, it can be easily observed that for the inverted hierarchy (IH) the specified ranges of $m_{0}, \theta_{14}$, and $\Delta m_{43}^{2}$, the effective

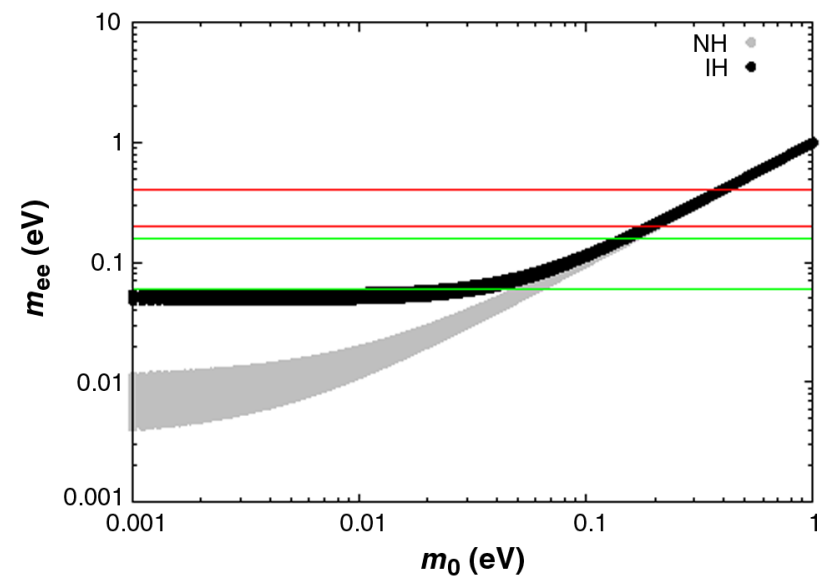

FIG. 5. The variation of the effective Majorana neutrino mass with the lightest neutrino mass for the normal and inverted hierarchies in the four-flavor (three active + one sterile) scenario. The pair of red lines and the pair of green lines indicate the limits obtained from different experiments (see text). For lower $m_{0}$ values, only the inverted hierarchy satisfies experimental limits. neutrino mass $m_{e e}$ is almost constant for smaller values of $m_{0}(0.001-0.01 \mathrm{eV})$. For higher values of $m_{0}, m_{e e}$ tends to increase proportionally with $m_{0}$. A similar trend is observed for the normal hierarchy $(\mathrm{NH})$ of neutrino masses when $m_{0} \geq 0.1 \mathrm{eV}$ is considered. However, for smaller values of $m_{0}(\leq 0.1 \mathrm{eV})$ the effective neutrino mass $m_{e e}$ in the case of normal hierarchy tends to decrease. The observed upper limit on the effective Majorana neutrino mass obtained from the combined analysis of KamLANDZen [70] and EXO-200 [71] is 0.2-0.4 eV, which corresponds to the region within the pair of red lines shown in Fig. 5. Therefore, in the above-specified range $\mathrm{NH}$ and $\mathrm{IH}$ are indistinguishable. A stringent limit on $m_{e e}$ is further obtained from KamLAND-Zen [72] (the region within the horizontal green lines in Fig. 5), with $m_{e e} \sim 0.06-0.16 \mathrm{eV}$ probing the near inverted hierarchy regime. From Fig. 5 it can be easily observed that the lightest neutrino mass $m_{0}$ must be larger than $0.1 \mathrm{eV}$ for higher values of $m_{e e}$. However, for the inverted hierarchy, the lightest neutrino mass $m_{0}$ can be smaller $(\sim 0.02 \mathrm{eV})$ when the limits on $m_{e e}$ from KamLAND [72] are taken into account. It is to be noted that in the present discussion we have neglected the Majorana phases. However, one should consider all of the Majorana phases. An extensive study of the effective neutrino mass including all of the Majorana phases has been presented in a recent work [73] using $\sin ^{2} \theta_{14}=0.019$ for $\Delta m_{41}^{2}=1.7 \mathrm{eV}^{2}$. For further details, see Ref. [73] and references therein.

\section{SUMMARY AND CONCLUSIONS}

We investigated the deviations of UHE neutrino signatures obtained from GRB events in a $1 \mathrm{~km}^{2}$ detector (such as IceCube) for a $3+1$ neutrino framework from the usual three-active-neutrino scenario. We considered a four-flavor scenario with three light active neutrinos and one sterile neutrino. The ratio of muon events to the shower events was calculated for both the three-flavor and four-flavor cases, which are denoted as $R_{3}$ and $R_{4}$. For this purpose, we have considered two sets of UHE neutrino fluxes. The first one is the theoretical flux for diffuse isotropic UHE neutrinos from GRBs given by Waxman and Bahcall, whereas the other was adopted from the analysis of the recent IceCube data. Using the present limits on active-sterile mixing obtained from different neutrino experiments along with the active neutrino mixing results, we found that the maximum value of the ratio of muon events with respect to shower events $R_{4}$ can be 6-8 times larger for the $3+1$ mechanism compared to that for the normal three active neutrino formalism $\left(R_{3}\right)$ if the Waxman-Bahcall flux is considered, and $R_{4}$ can be 3-4 times greater than $R_{3}$ using the flux given by the IceCube (HESE data) analysis. Therefore, the present analysis has shown that any excess of such events detected in a $1 \mathrm{~km}^{2}$ detector over that predicted for three-neutrino mixing can clearly indicate the presence of active-sterile neutrino mixing. Thus, UHE 
neutrinos from distant GRBs can be a probe to ascertain the existence of a sterile neutrino. In addition, we have also investigated neutrino-induced muon events from a single GRB in the present framework of $3+1$ neutrinos and compared the results with the three-flavor scenario. For a single GRB, with the observed bounds on active-sterile neutrino mixing, there is no significant deviation from threeactive-neutrino results. Therefore, for a single GRB, it is difficult to discriminate between the three- and four-flavor (three active + one sterile) formalisms. We further investigated the bounds on the light neutrino mass in the present four-neutrino scheme obtained from neutrinoless doublebeta decay search results. We estimated the order of the light neutrino mass for the normal hierarchy, using the present bounds on active-sterile mixing and the bounds from neutrinoless double-beta decay. We found that for the inverted hierarchy, the lightest neutrino mass can be as small as $\sim 0.02 \mathrm{eV}$ when bounds from KamLAND are considered.

There is room for systematic and detector-related uncertainties as well as the uncertainty in estimating the GRB neutrino flux. Indeed, the analysis of recent IceCube HESE data estimated the diffuse GRB neutrino flux as $E^{2} \phi(E)=$ $2.46 \pm 0.8 \times 10^{-8}\left(\frac{E}{100 \mathrm{TeV}}\right)^{-0.92} \mathrm{GeV} \mathrm{cm}^{-2} \mathrm{~s}^{-1} \mathrm{sr}^{-1}$. The uncertainty in the spectral index was also estimated to be $2.92_{-0.29}^{+0.33}$ in the same analysis. Uncertainties due to neutrino propagation and interactions inside the Earth, as well as detector and ice uncertainties can affect the observational data. Neutrino propagation inside the Earth is guided by the Earth density profile, which in this case was parametrized using PREM. In general, the uncertainty in PREM is considered by creating perturbations of the model. It is considered that the density gradient inside the Earth is negative in the core and mantle of the Earth.
The local perturbation in the PREM model is not generally taken to be more than $10 \%$. The shower and track events are recorded by digital optical modules (DOMs). Uncertainties can arise from the DOMs as well as the ice immediately surrounding the DOMs. The cables attached to the DOMs and unknown local optical conditions can affect the optical efficiency, which in turn imparts error to the event distribution and the detected energy. The systematic and statistical errors and optical scattering in the ice may affect the absorption coefficients. Even the glacial flows of ice can contribute to the uncertainty due to the ice. These uncertainties affect the light deposited by the LED flashers in the DOMs. A Monte Carlo analysis of such ice uncertainties shows a $\sim 10 \%$ variation [74]. The column of ice in the immediate vicinity of the DOMs may have different optical properties because of the possible trapping of gas during refreezing after their installation. This can introduce an additional uncertainty in recording the actual events. A detailed analysis and comparisons with the Monte Carlo simulations were discussed in Ref. [74]. When a quantity is obtained in terms of the ratios of two observables, such as the one (track-to-shower ratio) considered in the present work, some of the errors are expected to cancel.

\section{ACKNOWLEDGMENTS}

A. D. B. acknowledges the support from the Department of Science and Technology, Government of India under the fellowship reference number PDF/2016/002148 (SERB National Post-Doctoral fellowship). M. P. thanks the DST-INSPIRE fellowship grant (DST/INSPIRE Fellowship/[IF160004]) by DST, Govt. of India.
[1] C. Athanassopoulos et al. (LSND Collaboration), Phys. Rev. Lett. 77, 3082 (1996).

[2] A. Aguilar et al. (LSND Collaboration), Phys. Rev. D 64, 112007 (2001).

[3] J. M. Conard, W. C. Louis, and M. H. Shaevitz (LSND Collaboration), Annu. Rev. Nucl. Part. Sci. 63, 45 (2013).

[4] A. A. Aguilar-Arevalo et al. (MiniBooNE Collaboration), Phys. Rev. Lett. 98, 231801 (2007).

[5] A. A. Aguilar-Arevalo et al. (MiniBooNE Collaboration), Phys. Rev. Lett. 105, 181801 (2010).

[6] T. A. Mueller et al., Phys. Rev. C 83, 054615 (2011).

[7] P. Huber, Phys. Rev. C 84, 024617 (2011).

[8] G. Mention, M. Fechner, Th. Lasserre, Th. A. Mueller, D. Lhuillier, M. Cribier, and A. Letourneau, Phys. Rev. D 83, 073006 (2011).

[9] P. Anselman et al. (GALLEX Collaboration), Phys. Lett. B 342, 440 (1995).
[10] W. Hampel et al. (GALLEX Collaboration), Phys. Lett. B 420, 114 (1998).

[11] J. N. Abdurashitov et al., Phys. Rev. Lett. 77, 4708 (1996).

[12] J. N. Abdurashitov et al. (SAGE Collaboration), Phys. Rev. C 59, 2246 (1999).

[13] J. N. Abdurashitov et al., Phys. Rev. C 73, 045805 (2006).

[14] P. Adamson et al. (MINOS Collaboration), Nucl. Instrum. Methods Phys. Res., Sect. A 806, 279 (2016).

[15] P. Adamson et al. (MINOS Collaboration), Phys. Rev. Lett. 117, 151803 (2016).

[16] P. Adamson et al. (MINOS Collaboration), arXiv:1710.06488.

[17] P. Adamson et al. (MINOS Collaboration), Phys. Rev. Lett. 110, 171801 (2013).

[18] P. Adamson et al. (MINOS Collaboration), Phys. Rev. Lett. 107, 011802 (2011)

[19] D. G. Michael et al. (MINOS Collaboration), Phys. Rev. Lett. 97, 191801 (2006). 
[20] P. Adamson et al. (MINOS Collaboration), Phys. Rev. Lett. 101, 221804 (2008).

[21] P. Adamson et al. (MINOS Collaboration), Phys. Rev. Lett. 112, 191801 (2014).

[22] P. Adamson et al. (MINOS Collaboration), Phys. Rev. D 81, 052004 (2010).

[23] D. G. Michael et al. (MINOS Collaboration), Nucl. Instrum. Methods Phys. Res., Sect. A 596, 190 (2008).

[24] P. Adamson et al. (MINOS Collaboration), Phys. Rev. D 77, 072002 (2008).

[25] P. Adamson et al. (Daya Bay and MINOS Collaborations), Phys. Rev. Lett. 117, 151801 (2016).

[26] F. P. An et al. (Daya Bay Collaboration), Phys. Rev. Lett. 108, 171803 (2012).

[27] F. P. An et al. (Daya Bay Collaboration), Phys. Rev. Lett. 117, 151802 (2016).

[28] F. P. An et al. (Daya Bay Collaboration), Nucl. Instrum. Methods Phys. Res., Sect. A 811, 133 (2016).

[29] F. P. An et al. (Daya Bay Collaboration), Phys. Rev. Lett. 112, 061801 (2014).

[30] F. P. An et al. (Daya Bay Collaboration), Phys. Rev. Lett. 116, 061801 (2016).

[31] F. P. An et al. (Daya Bay Collaboration), Phys. Rev. Lett. 115, 111802 (2015).

[32] F. P. An et al. (Daya Bay Collaboration), Phys. Rev. Lett. 113, 141802 (2014).

[33] B. Achkar et al. (BUGEY Collaboration), Nucl. Phys. B434, 503 (1995).

[34] R. Acciari et al. (DUNE Collaboration), arXiv:1512.06148.

[35] R. Acciari et al. (DUNE Collaboration), arXiv:1601.02984.

[36] R. Acciari et al. (DUNE Collaboration), arXiv:1601.05471.

[37] J. Strait et al. (DUNE Collaboration), arXiv:1601.05823.

[38] T. Ishida (for the Hyper-Kamiokande Working Group), arXiv:1311.5287.

[39] Y. Abe, Y. Asano, N. Haba, and T. Yamada, Eur. Phys. J. C 77, 851 (2017).

[40] S. Choubey, D. Dutta, and D. Pramanik, Phys. Rev. D 96, 056026 (2017).

[41] P. Coloma, D. V. Forero, and S. J. Parke, Report No. FERMILAB-PUB-17-271-T (2017), arXiv:1707.05348.

[42] J. Ahrens et al. (IceCube Collaboration), in Proceedings of the 27th International Cosmic Ray Conference, Hamburg, Germany, 2001 (unpublished), p. 1237, http://icecube.wisc .edu.

[43] S. Dado, A. Dar, and A. D. Rujula, Astron. Astrophys. 388, 1079 (2002).

[44] A. Dar and A. De Rujula, Phys. Rep. 405, 203 (2004).

[45] A. Dar, Chin. J. Astron. Astrophys. 6, 301 (2006).

[46] S. Dado and A. Dar, AIP Conf. Proc. 1111, 333 (2009).

[47] D. Majumdar and A. Ghosal, Phys. Rev. D 75, 113004 (2007).

[48] Z. Maki, M. Nakagawa, and S. Sakata, Prog. Theor. Phys. 28, 870 (1962).
[49] S. K. Kang, Y.-D. Kim, Y.-J. Ko, and K. Siyeon, Adv. High Energy Phys. 2013, 138109 (2013).

[50] H. Athar, M. Jezabek, and O. Yasuda, Phys. Rev. D 62 , 103007 (2000).

[51] E. Waxman and J. Bahcall, Phys. Rev. Lett. 78, 2292 (1997).

[52] E. Waxman and J. Bahcall, Phys. Rev. Lett. 59, 023002 (1998).

[53] R. Gandhi, C. Quigg, M. H. Reno, and I. Sarcevic, Phys. Rev. D 58, 093009 (1998).

[54] R. Gandhi, C. Quigg, M. H. Reno, and I. Sarcevic, Astropart. Phys. 5, 81 (1996).

[55] T. K. Gaisser, Cosmic Rays and Particle Physics (Cambridge University Press, Cambridge, England, 1992); T. K. Gaisser, F. Halzen, and T. Stanev, Phys. Rep. 258, 173 (1995).

[56] N. Gupta, Phys. Rev. D 65, 113005 (2002).

[57] A. Dziewonski, Earth structure, global, in The Encyclopedia of Solid Earth Geophysics, edited by D. E. James (Van Nostrand Reinhold, New York, 1989), p. 331.

[58] A. Dar, J. J. Lord, and R. J. Wilkes, Phys. Rev. D 33, 303 (1986).

[59] D. Guetta, D. Hooper, J. A. Muniz, F. Halzen, and E. Reuveni, Astropart. Phys. 20, 429 (2004).

[60] S. I. Dutta, M. H. Reno, and I. Saecevic, Phys. Rev. D 62 , 123001 (2000).

[61] N. Gupta, Phys. Lett. B 541, 16 (2002).

[62] P. Adamson et al. (NOvA Collaboration), Phys. Rev. D 96, 072006 (2017).

[63] D. S. Ayres et al. (NOvA Collaboration), arXiv:hep-ex/ 0503053.

[64] P. Adamson et al. (NOvA Collaboration), Phys. Rev. Lett. 116, 151806 (2016).

[65] P. Adamson et al. (NOvA Collaboration), Phys. Rev. Lett. 118, 231801 (2017).

[66] P. Adamson et al. (NOvA Collaboration), Phys. Rev. D 93, 051104(R) (2016).

[67] P. Adamson et al. (NOvA Collaboration), Phys. Rev. Lett. 118, 151802 (2017).

[68] M. G. Aartsen et al. (IceCube Collaboration), Phys. Rev. D 95, 112002 (2017).

[69] IceCube Collaboration, Proc. Sci., ICRC2017 (2017) 981.

[70] A. Gando et al. (KamLAND-Zen Collaboration), Phys. Rev. Lett. 110, 062502 (2013).

[71] J. B. Albert et al. (EXO-200 Collaboration), Nature (London) 510, 229 (2014).

[72] A. Gando et al. (KamLAND-Zen Collaboration), Phys. Rev. Lett. 117, 082503 (2016).

[73] J. H. Liu and S. Zhou, Int. J. Mod. Phys. A 33, 1850014 (2018).

[74] M. G. Aartsen et al. (IceCube Collaboration), Phys. Rev. Lett. 117, 071801 (2016). 